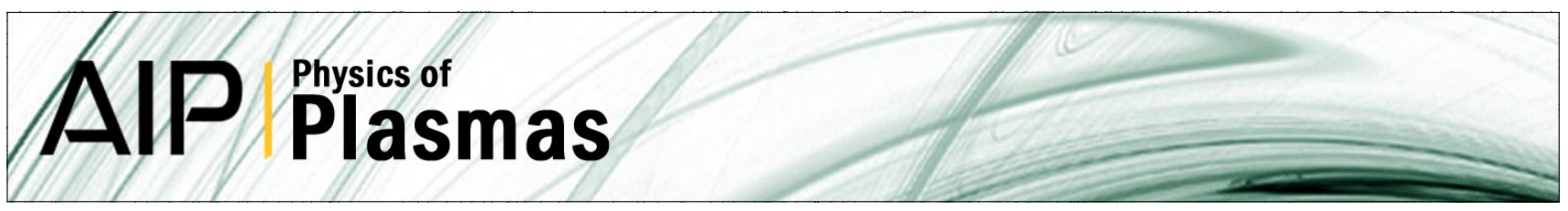

\title{
Coalescence of two magnetic flux ropes via collisional magnetic reconnection
}

Ivo Furno, Thomas P. Intrator, Erik W. Hemsing, Scott C. Hsu, S. Abbate et al.

Citation: Phys. Plasmas 12, 055702 (2005); doi: 10.1063/1.1894418

View online: http://dx.doi.org/10.1063/1.1894418

View Table of Contents: http://pop.aip.org/resource/1/PHPAEN/v12/i5

Published by the American Institute of Physics.

\section{Related Articles}

Morphology and dynamics of three interacting kink-unstable flux ropes in a laboratory magnetoplasma Phys. Plasmas 19, 102102 (2012)

Magnetic reconnection in space

Phys. Plasmas 19, 092902 (2012)

Spectroscopic observation of simultaneous bi-directional reconnection outflows in a laboratory plasma Phys. Plasmas 19, 080704 (2012)

The role of compressibility in energy release by magnetic reconnection

Phys. Plasmas 19, 082109 (2012)

Design of a dual sensor probe array for internal field measurement in Versatile Experiment Spherical Torus Rev. Sci. Instrum. 83, 10D721 (2012)

\section{Additional information on Phys. Plasmas}

Journal Homepage: http://pop.aip.org/

Journal Information: http://pop.aip.org/about/about_the_journal

Top downloads: http://pop.aip.org/features/most_downloaded

Information for Authors: http://pop.aip.org/authors

\section{ADVERTISEMENT}

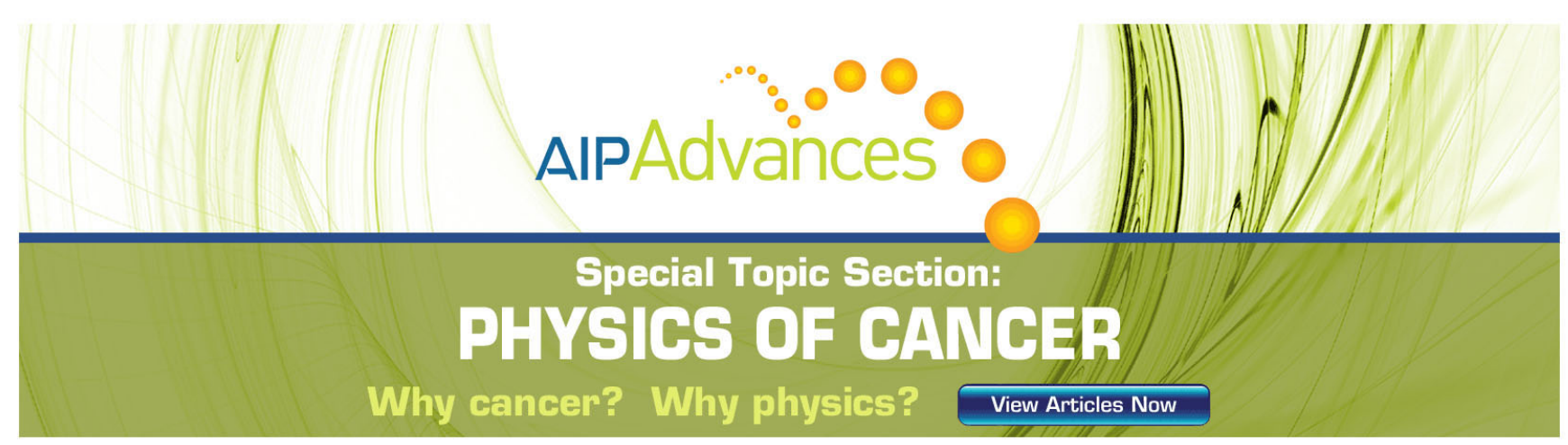




\title{
Coalescence of two magnetic flux ropes via collisional magnetic reconnection ${ }^{\text {a) }}$
}

\author{
Ivo Furno, ${ }^{\text {b) }}$ Thomas P. Intrator, ${ }^{c)}$ Erik W. Hemsing, Scott C. Hsu, and S. Abbate \\ Los Alamos National Laboratory, M.S. E526, Los Alamos, New Mexico 87545 \\ Paolo Ricci \\ Department of Physics and Astronomy, Dartmouth College, Hanover, New Hampshire 03755
}

\begin{abstract}
Giovanni Lapenta
Istituto Nazionale per la Fisica della Materia (INFM), Unità del Politecnico di Torino, Corso Duca degli Abruzzi 24 -10129 Torino, Italy and Los Alamos National Laboratory, Los Alamos, New Mexico 87545
\end{abstract}

(Received 19 November 2004; accepted 14 February 2005; published online 5 May 2005)

\begin{abstract}
Quasi-two-dimensional coalescence of two parallel cylindrical flux ropes and the development of three-dimensional merged structures are observed and studied in the reconnection scaling experiment [Furno et al., Rev. Sci. Instrum. 74, 2324 (2003)]. These experiments were conducted in a collisional regime with very strong guide magnetic field ( $\left.B_{\text {guide }} \gg B_{\text {reconnection }}\right)$, which can be adjusted independently of plasma density, current density, and temperature. During initial coalescence, a reconnection current sheet forms between the two flux ropes, and the direction of the current is opposite to the flux rope currents. The measured current sheet thickness is larger than the electron skin depth but smaller than the ion skin depth. Furthermore, the thickness does not vary for three different values of the strong external guide field. It is shown that the geometry of the observed current sheet is consistent with the Sweet-Parker model using a parallel Spitzer resistivity. The flux ropes eventually become kink unstable due to increasing current and fast-gated camera images show the development of three-dimensional merged structures. (c) 2005 American Institute of Physics.

[DOI: $10.1063 / 1.1894418$ ]
\end{abstract}

\section{INTRODUCTION}

Magnetic fields are embedded in astrophysical, solar, space, and laboratory plasmas. For many of these cases, the magnetic field is organized in structures called flux tubes and/or flux ropes. ${ }^{1}$ In this work, we define a magnetic flux rope as a structure in which the magnetic field lines are twisted, implying that field-aligned currents are present. When two flux ropes are close enough, they interact. The dynamics and relaxation of closely interacting flux ropes can involve flows, changes in field topology, dissipation of magnetic energy, magnetic reconnection, and plasma heating. Nature provides numerous examples of dynamic flux rope interactions including the evolution of solar flares, ${ }^{2}$ dynamics of the Earth's magnetosphere, ${ }^{3,4}$ and possibly evolution of astrophysical plasmas, such as galactic jets. ${ }^{5,6}$ In magnetically confined laboratory plasmas for fusion research, flux rope interactions can determine the dynamics of relaxation processes, such as sawtooth oscillations in tokamaks, ${ }^{7}$ and spheromak formation. ${ }^{8}$

Recently, laboratory experiments in both toroidal ${ }^{9-13}$ and linear geometry ${ }^{14}$ together with numerical simulations ${ }^{15-17}$ have highlighted the importance of three-dimensional (3D) effects in the dynamics of flux rope evolution.

In the reconnection scaling experiment ${ }^{18}(\mathrm{RSX})$ at Los

\footnotetext{
a) Paper NI2 2, Bull. Am. Phys. Soc. 49, 249 (2004).

b)Invited speaker. Electronic mail: furno@lanl.gov

c)Electronic mail: intrator@lanl.gov
}

Alamos National Laboratory, we take advantage of recently developed plasma gun technology ${ }^{19}$ to create parallel flux ropes and to study their interaction in a linear geometry that supports fully 3D evolution. The RSX axial guide field $\left(B_{0 z}=0-2 \mathrm{kG}\right)$ can be adjusted independently of the plasma density, temperature, and current density. This independent scalability of the ion gyroradius thus allows parametric scans of plasma $\beta$.

In this paper, we study the coalescence of twin flux ropes in the limit of very large guide field. We show that a current sheet develops between the flux ropes during the coalescence process. The thickness of the current sheet and the maximum current density do not depend on the guide field, and the thickness is consistent with the Sweet-Parker model ${ }^{20,21}$ using a parallel Spitzer plasma resistivity.

The remainder of the paper is organized as follows. In Sec. II, the experimental setup and diagnostics are described. In Sec. III, we present and discuss experimental measurements during the interaction of two flux ropes in the presence of a strong guide field. Extensions of this work are suggested in Sec. IV and a summary follows in Sec. V.

\section{EXPERIMENT AND PLASMA DESCRIPTION}

\section{A. RSX device and plasma properties}

A view of the RSX device is shown in Fig. 1(a) together with a schematic of the linear geometry of two flux ropes. The RSX has a cylindrical vacuum chamber with approxi- 
mately $4 \mathrm{~m}$ length and $0.2 \mathrm{~m}$ radius and, for the experiments presented here, is equipped with two plasma guns ${ }^{19}$ radially inserted from top and bottom as shown in Fig. 1. The radial distance between plasma guns can be modified to adjust the distance between the interacting flux ropes. Each plasma gun contains a miniaturized plasma source, Fig. 1(b), with a circular $0.79 \mathrm{~cm}^{2}$ nozzle aperture in which a hydrogen plasma is produced by an arc discharge between a molybdenum anode and cathode. The arc plasma is maintained for $\approx 10 \mathrm{~ms}$ by a pulse forming network which is energized by a capacitor bank charged up to $V_{\text {arc }}<1 \mathrm{kV}$. The arc current $I_{\text {arc }}$ rapidly increases and reaches a maximum value of about $1 \mathrm{kA}$ just before the external anode is biased. The pulse length is limited only by the gas inventory inside the gun volume and the pulse forming network. The plasma emitted from the plasma guns is immersed in a constant, uniform, axial (in the $z$ direction) background magnetic field $B_{0 z}$, which can be as large as $2 \mathrm{kG}$.

A flux rope is created in the gun plasma by driving a current between the gun anode and an external anode as schematically shown in Fig. 1(b). The external anode is a $1000 \mathrm{~cm}^{2}$ SS304 plate which is electrically insulated from the vacuum vessel. Its axial location along the RSX vessel determines the length of the flux ropes. A fraction $\left(I_{\text {bias }}\right)$ of the arc current is diverted from each gun, where the electron current direction is towards the external anode. The bias discharge is initiated approximately $1 \mathrm{~ms}$ after the beginning of the arc discharge, corresponding to the maximum of $I_{\text {arc }}$. Driven plasma current steadily increases after the bias is switched on as shown in Fig. 1(c). The applied bias voltage and the inductance of the total circuit (including the plasma) determine the slope of the applied current ramp [typically $\left.d I_{\text {bias }} / d t \approx(10-20) \times 10^{6} \mathrm{~A} / \mathrm{s}\right]$.

For the gun generated flux ropes and reconnection regions in between them, typical density is $n_{e} \approx(0.4-5)$ $\times 10^{13} \mathrm{~cm}^{-3}$, electron temperature is $k_{B} T_{e}<7 \mathrm{eV}$ early in time for $t<15 \mu \mathrm{s}$ and $k_{B} T_{e}<12 \mathrm{eV}$ later in time for $t$ $>15 \mu$ s (the Boltzman constant $k_{B}$ will be suppressed hereafter). The reconnection region scale size is $\Delta \approx 2 \mathrm{~cm}$, Spitzer parallel resistivity $\eta_{\|} \approx 30-150 \mu \Omega \mathrm{m}$. The reconnection magnetic fields are on the order of $B_{1 x} \approx 2-10 \mathrm{G}$, the derived Alfvén speeds are approximately $v_{A} \approx(1-6)$ $\times 10^{5} \mathrm{~cm} / \mathrm{s}$. The Lundquist number defined as $S$ $=\Delta v_{A} /\left(2 \eta_{\|} / \mu_{0}\right)$ is in the range $S \approx 0.5-10$. The plasma downstream of the gun is typically $30 \%-80 \%$ ionized, while ionization is nearly complete near the gun nozzle. Electronneutral collision rates are far less than electron-ion collision rates and thus are neglected in this work. ${ }^{18}$

\section{B. Diagnostics}

This experimental plasma has low enough temperature $\left(T_{e}<20 \mathrm{eV}\right)$ and short enough pulse length $(<10 \mathrm{~ms})$ to enable in situ measurements using internal probes. Provided that the plasma dynamics are reproducible, fine spatial resolution in the $x-y$ plane is achieved by moving internal probes between RSX discharges. Probe data from different axial ports allows assembly of 3D measurements at various $z$ locations.
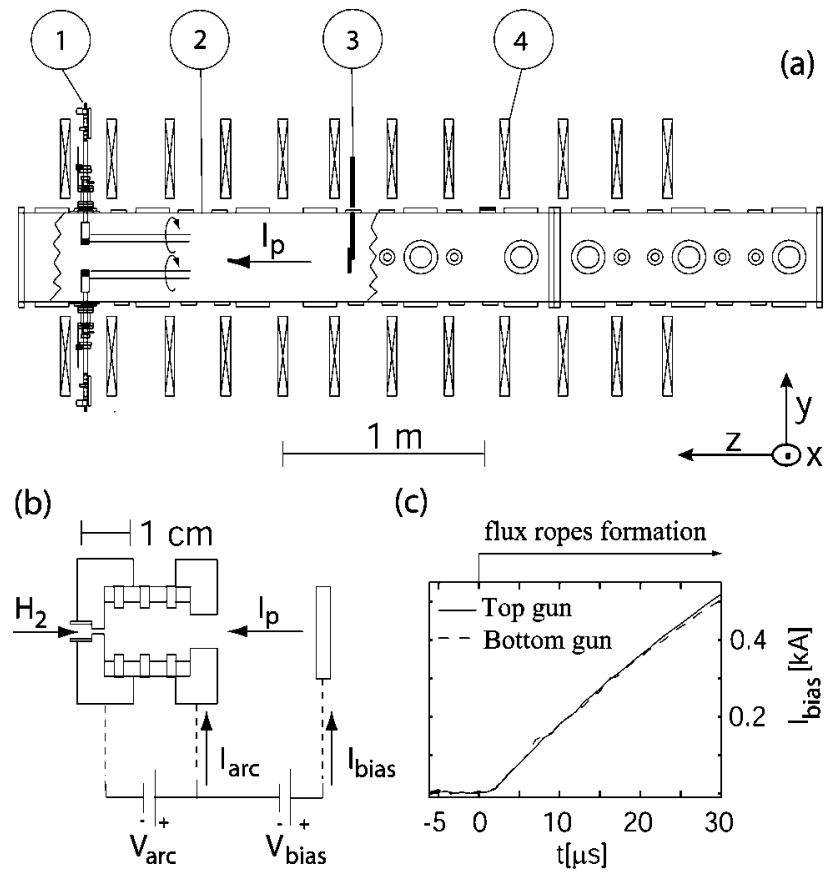

FIG. 1. RSX experimental configuration. (a) Side view of RSX with (1) plasma guns, (2) vacuum vessel, (3) external anode, (4) external magnet coils, schematic of two flux ropes with their magnetic fields, and the coordinate system. Each flux rope current flows in the positive $z$ direction. (b) Expanded view of the miniaturized plasma injector with main circuit elements (not to scale). (c) Time history of gun bias currents during the formation of two flux ropes.

Magnetic field structures are measured using miniaturized, two-dimensional magnetic probe arrays with ten pickup coils (each with a total equivalent area turns of $3 \times 10^{-4} \mathrm{~m}^{2}$ ) equispaced by $2.5 \mathrm{~mm}$. Two adjacent coils measure magnetic fields in orthogonal directions in space. Measurements in the third spatial direction can be obtained by rotating the probe. The signals are digitized at $20 \mathrm{MHz}$. Electron density $n_{e}$, temperature $T_{e}$, and pressure $p_{e}=n_{e} T_{e}$ are simultaneously measured using a movable triple electrostatic probe with a 1 $\mathrm{mm}$ tip separation.

Visible light (mostly $H_{\alpha}$ at $656.3 \mathrm{~nm}$ ) emitted from the plasma is monitored with a Cooke DiCam Pro intensified charge-coupled device camera. ${ }^{22,23}$ The viewing geometry of the camera is schematically shown in Fig. 8 below. The camera provides two 12-bit images per shot, each with 1280 $\times 1024$ pixel resolution. In the present setup, Alfvén times $\tau_{A}=\Delta / v_{A} \approx 0.7-1 \mu \mathrm{s}$ are estimated to be long compared to gate times of 40-200 ns, so the images should accurately resolve the plasma dynamics. ${ }^{22}$

\section{EXPERIMENTAL RESULTS}

In this section, we show the first laboratory measurements of flux rope coalescence in a linear geometry and in the presence of a strong axial guide field. We discovered that RSX discharges are highly reproducible during the first $15 \mu$ s after the drive has started. Magnetic data and particle pressure contours are shown in Sec. III A that lead to the conclusion that magnetic reconnection occurs between the flux ropes. The characterization of Sweet-Parker-like $20,21,24$ 

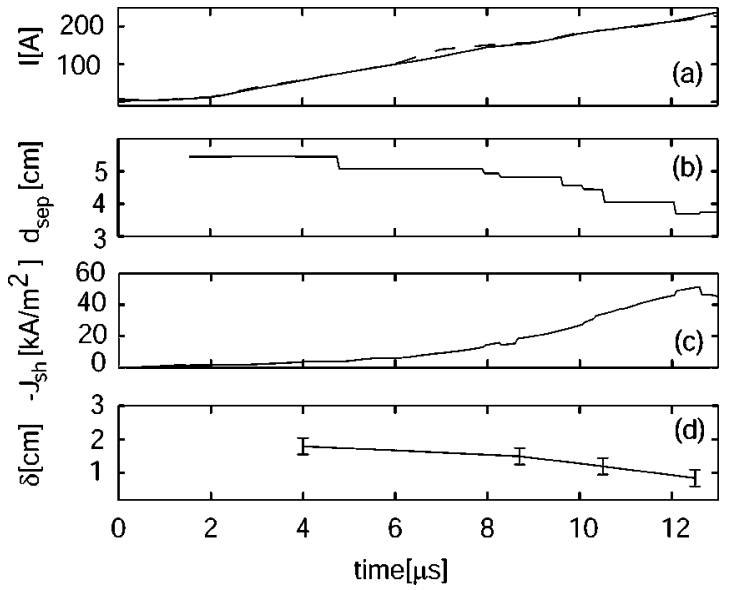

FIG. 2. Time history of plasma parameters during the interaction of two flux ropes. (a) Bias current driven at each gun (top gun, dashed line; bottom gun, solid line). (b) Distance between flux rope centers $d_{\text {sep }}$. (c) Peak current density driven in the current sheet. (d) Thickness of the current sheet $\delta$.

current sheet in this region is explored in Sec. III B. For later times, the magnetic dynamics of flux rope coalescence are less reproducible from shot-to-shot and in Sec. III C multiple fast images of plasma light are used to gain insight into the development of helical structures and 3D dynamics. For these experiments the gun parameters were $V_{\mathrm{arc}}=510 \mathrm{~V}$, $I_{\text {arc }}=0.8 \mathrm{kA}, P_{\text {gas }}=20 \mathrm{psi}$. Unless otherwise specified, the background magnetic field was $B_{0 z}=200 \mathrm{G}$. The two guns are spaced approximately $5.5 \mathrm{~cm}$ apart along a diameter at $z=0$ and the external anode is located at $z=+0.9 \mathrm{~m}$.

\section{A. Coalescence of flux ropes}

In this section, we show evidence that magnetic reconnection occurs in coalescing region between the two flux ropes, while they are attracted to each other by $\mathbf{J} \times \mathbf{B}$ forces. This is a strong drive that we believe compresses the plasma in between the current channels as well as the embedded magnetic flux.

In Fig. 2(a), the time evolution of the bias currents for both guns is shown during the first $13 \mu$ s of the experiment. The flux rope formation is initiated at $t=0$ when the arc anode is biased to $V_{\text {bias }}=+200 \mathrm{~V}$ with respect to the external anode. Figure 3 shows the projection of the magnetic field in the in the $x-y$ plane (see Fig. 1), $\mathbf{B}_{\perp}=B_{x} \mathbf{e}_{x}+B_{y} \mathbf{e}_{y}$. These data are assembled over $40 \mathrm{RSX}$ discharges with a $5 \mathrm{~mm}$ spatial resolution. The four frames correspond to four different times spaced by $3 \mu$ s during the current ramp up. Color contours indicate axial current density $J_{z}$, and green continuous lines denote constant vector potential $A_{z}$ corresponding to magnetic field lines $\left(\mathbf{B}_{\perp}=\boldsymbol{\nabla} \times A_{z}\right)$. Current density and vector potential are computed, respectively, from $\mu_{0} J_{z} e_{z}=\nabla$ $\times \mathbf{B}_{\perp}$ and $\nabla^{2} A_{z}=\mu_{0} J_{z}$.

The axial current flows in each flux rope is in the positive $z$ direction (from the external anode to the guns) and the magnetic fields in between them are antiparallel. Both flux ropes have the same helicity (cohelicity), since $B_{0 z}$ has the same sense relative to $J_{z}$ for each flux rope. The twist of each

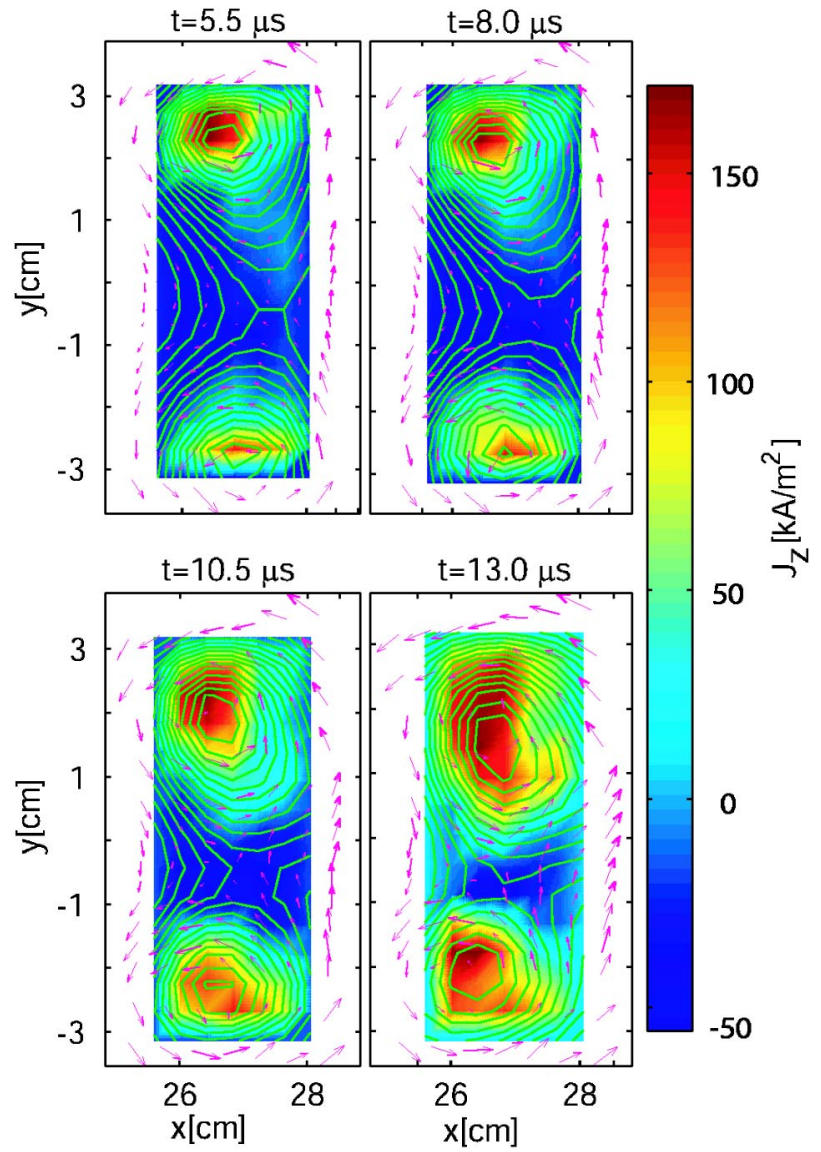

FIG. 3. (Color online) Structures of the transverse magnetic field $\mathbf{B}_{\perp}(x, y)$ during the interaction of twin flux ropes at the different times during the current ramp up. Magnetic field lines corresponding to lines of constant vector potential $A_{z}$ are also shown as continuous lines.

flux rope steadily increases in time, but the external kink mode threshold for stability is not exceeded for this early phase. $^{25}$

Early in time, the magnetic field $\mathbf{B}_{\perp}$ acquires an $X$-point topology near the geometric center of the two flux ropes, as shown in Fig. 3. The separatrix occurs at right angles, satisfying approximately $\boldsymbol{\nabla} \times \mathbf{B}_{\perp}=0$ and no current at the $X$-point is driven.

Later in time, mutually attractive $\mathbf{J} \times \mathbf{B}$ forces decrease the separation distance as shown in Fig. 2(c), where $d_{\text {sep }}$ denotes the distance between positions of maximum $J_{z}$ in each flux rope. The closing velocity is a fraction of the Alfvén speed defined using $B_{\perp}$. As the two flux ropes approach each other, the magnetic flux is compressed, the field lines flatten near the neutral region and a reversed current is induced in the negative $z$ direction. Figures 3(b)-3(d) show a local sheet structure of width $\Delta$ which evolves from $\Delta$ $\approx 1.7 \mathrm{~cm}$ at $t=5.5 \mu$ s to $\Delta \approx 2 \mathrm{~cm}$ at $t=13 \mu \mathrm{s}$. Here, we define $\Delta$ as the distance between locations along the line perpendicular to the center-to-center line where $J_{z}=1 / 4 J_{\mathrm{sh}}$, where $J_{\text {sh }}$ is the minimum current density in the current sheet. At these locations, magnetic field lines are approximately parallel to the center-to-center line. The time evolution of $J_{z}$ along the center-to-center line is shown in Fig. 4. $J_{z}$ peaks in each flux rope, and the reversed current sheet has a 

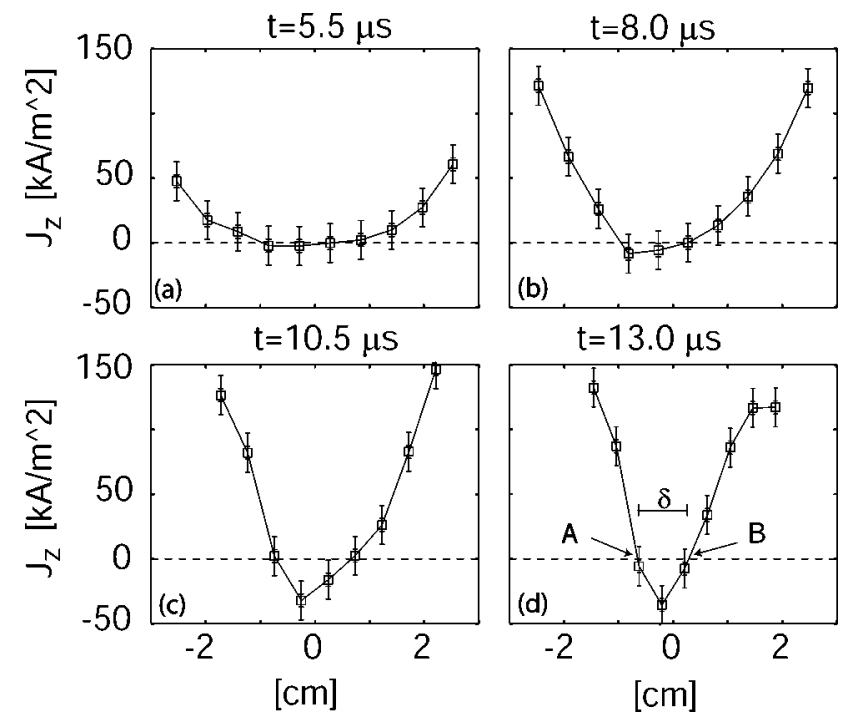

FIG. 4. Axial current density $J_{z}$ at $z=50 \mathrm{~cm}$ evaluated along the line that connects the centers of the two flux ropes. Different times (same as in Fig. 3) are shown during the current ramp up. Positive current is driven in each flux rope, which induces a negative current sheet during the coalescence process. We define the thickness of the current sheet $\delta$ as the distance between points where $J_{z}=0$, e.g., points $A$ and $B$ in frame (d).

width $\delta$ we define as the distance between $J_{z}=0$ locations along the center-to-center line. The time histories of the peak $J_{\text {sh }}$ in the current sheet and $\delta$ are shown respectively in Figs. 2(c) and 2(d). The current sheet compresses in time to a thickness $\delta=\sim 0.8 \mathrm{~cm}$ which corresponds to a value between the electron skin depth $c / \omega_{\mathrm{pe}} \approx 2 \mathrm{~mm}$ and the ion skin depth $c / \omega_{\mathrm{pe}} \approx 4 \mathrm{~cm}$. This value of $\delta$ represents an upper limit, since the measured width is broadened by the spatial resolution of the magnetic probe $(5 \mathrm{~mm})$ array and also by averaging over fluctuations from shot to shot.

The plasma electron pressure contours also show the movements of the current channels, and are consistent with vector potential and current density data shown in Fig. 3. Figure 5 shows electron pressure $p_{e}=n_{e} T_{e}$ contours in the $x-y$ plane at $z=0.5 \mathrm{~m}$ from the $z=0$ plane of the two guns. Both for early times as the flux ropes form, and later in time as the merging and reconnection takes place. The flux rope profiles indicate a center electron temperature of $T_{e 0} \approx 7 \mathrm{eV}$ and center electron density of $n_{e 0} \approx 2.5 \times 10^{13} \mathrm{~cm}^{-3}$. The shape has a full width half maximum of $\approx 3 \mathrm{~cm}$. In the current sheet, the profiles indicate a peak electron temperature of $T_{e, \mathrm{sh}} \approx 2-4 \mathrm{eV}$ and density of $n_{e, \mathrm{sh}} \approx(0.5-3) \times 10^{13} \mathrm{~cm}^{-3}$.

\section{B. Current sheet characteristics}

This section shows the first experimental evidence of a Sweet-Parker reconnection layer in the presence of a strong guide field.

We take advantage of one RSX experimental capability to continuously adjust the axial guide field $\left(B_{0 z}=0-2 \mathrm{kG}\right)$ independently of the plasma density, temperature, and current density. To test the effect of the background guide field on the current layer geometry and coalescence dynamics, $B_{0 z}$ was varied by a factor of 4 , while the other conditions were kept constant, including $d I_{\text {bias }} / d t$, gun current, and density.

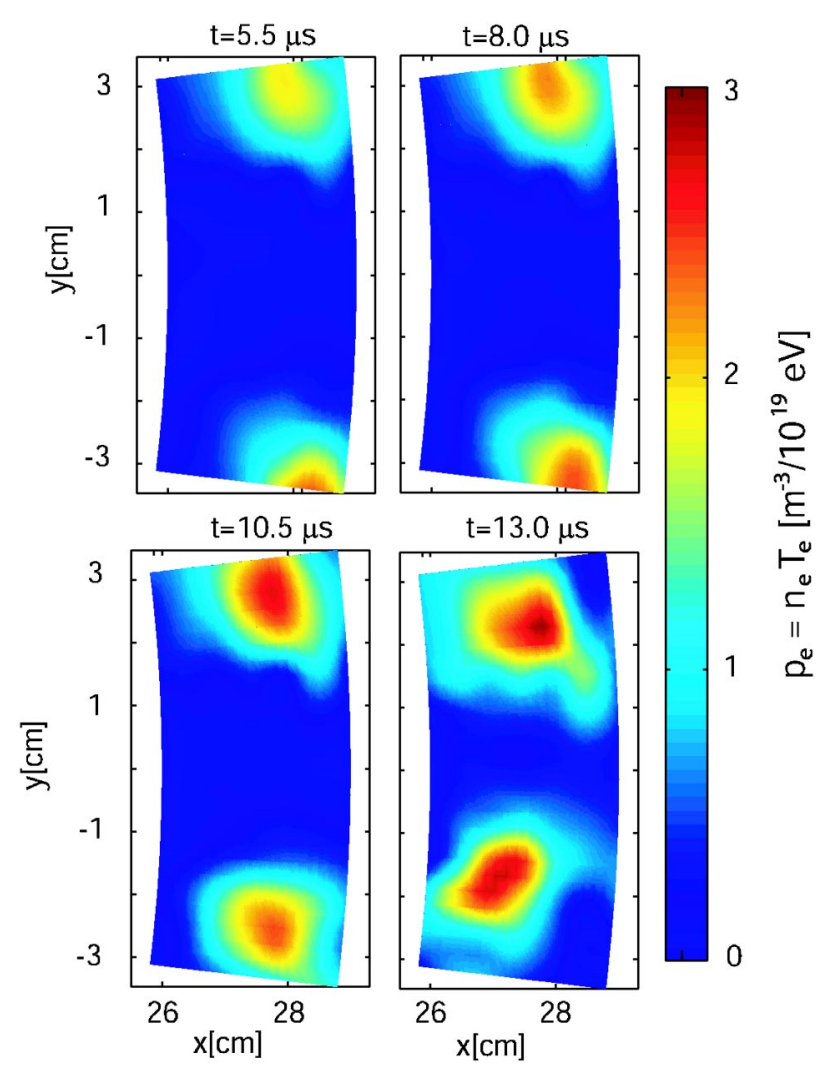

FIG. 5. (Color online) Contours of electron pressure at four times that encompass the creation and merging of two flux ropes. Data were obtained by a movable triple electrostatic probe (100 RSX discharges) in the $x-y$ plane perpendicular to the axis of the device $0.5 \mathrm{~m}$ from the two plasma guns, background $B_{0 z}=200 \mathrm{G}$.

Figure 6 shows some results from this set of experiments. The current sheet width $\delta$ and the peak current density in the center of the current sheet are shown for the three different values of axial magnetic field $\left(B_{0 z}=100,200,400\right.$ $\mathrm{G})$. This corresponds to a ratio of guide field to reconnection field $B_{\text {rec }}$ of approximately $B_{0 z} / B_{\text {rec }} \approx 10-40$, where $B_{\text {rec }}$ is the magnetic field at the edge of the current sheet evaluated as the component of $\mathbf{B}_{\perp}$ perpendicular to the center-to-center line. In the three cases, as a result of magnetic flux annihilation, a current sheet forms with a width smaller than the ion skin depth $c / \omega_{\mathrm{pi}}$, which for this set of experiments is $\approx 4 \mathrm{~cm}$. Figures 6(a) and 6(b) are consistent with each other in this picture. Neither the current sheet width nor the flux rope speed of approach seem to depend on $B_{0 z}$.

The two-dimensional Sweet-Parker reconnection model $^{20,21}$ can be easily derived from the plasma continuity equation, the equation of motion, and the induction equation which can be, respectively, written as

$$
\begin{aligned}
& \frac{\partial n}{\partial t}+\boldsymbol{\nabla} \cdot(n \mathbf{V})=0, \\
& \rho\left(\frac{\partial}{\partial t}+\mathbf{V} \cdot \boldsymbol{\nabla}\right) \mathbf{V}=-\boldsymbol{\nabla} p+\mathbf{J} \times \mathbf{B},
\end{aligned}
$$




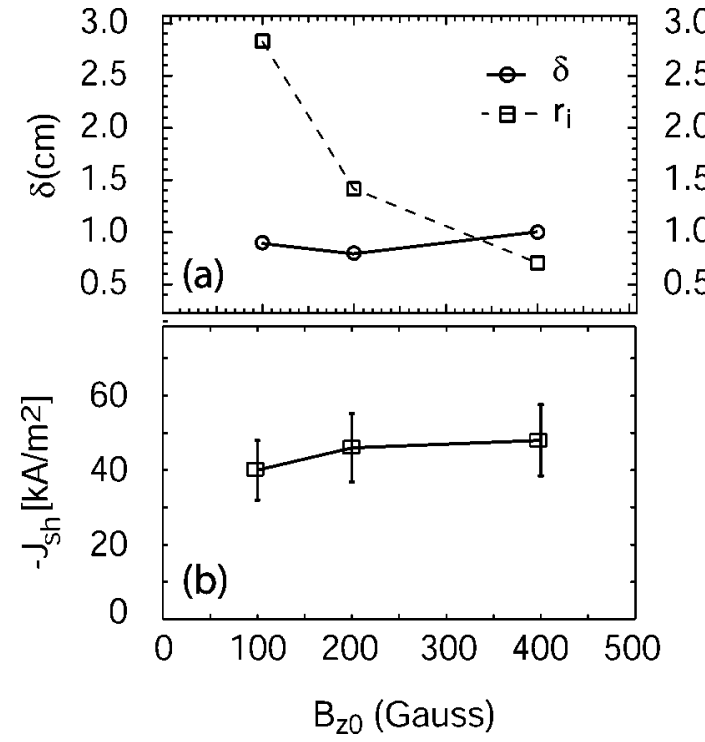

FIG. 6. Current sheet width and current density at different values of axial magnetic field, evaluated at the time of peak layer compression $t \approx 13 \mu \mathrm{s}$. (a) Current sheet thickness (circles, left-hand axis) together with the estimated ion gyroradius (squares, right-hand axis). (b) Reversed peak current density in the center of the current sheet.

$$
\frac{\partial \mathbf{B}}{\partial t}=\boldsymbol{\nabla} \times(\mathbf{V} \times \mathbf{B})+\frac{\eta}{\mu_{0}} \nabla^{2} \mathbf{B},
$$

where $\mathbf{V}$ is the magnetohydrodynamics flow velocity, $n$ is plasma density, and $\rho$ is the mass density.

In our set of experiments, the following assumptions hold: plasma incompressibility $(\partial n / \partial t \simeq 0)$, steady state plasma flow $(\partial \mathbf{V} / \partial t \simeq 0)$, uniform pressure outside the current sheet. Negligible quantities are $\rho V_{y}^{2} / 2$ in the inflow region, $B_{x}^{2} / 2 \mu_{0}$ in the outflow region, and tension forces. Equations (1) and (2) can be easily reduced to $v_{\text {out }}=V_{A}$, where $V_{A} \equiv B / \sqrt{\mu_{0} \rho}$ is the Alfvén speed, and $v_{\text {in }}=v_{\text {out }} \delta / \Delta$ (see inset in Fig. 7). The current sheet aspect ratio $\delta / \Delta$ can be estimated from Eq. (3) using the previous results. Compared with the original Sweet-Parker model, the assumption $\partial \mathbf{B} / \partial t=0$ is not strictly satisfied in our experiment. Retaining this term in Eq. (3) leads to the following expression:

$$
\frac{\delta}{\Delta}=\frac{1}{\sqrt{S}} \sqrt{1+\frac{\tau_{A}}{\tau_{B}} \frac{\delta}{2 \Delta} S}
$$

where $\tau_{A} \equiv \delta / V_{A}, \tau_{B} \equiv\left[\left(\partial B_{x} / \partial t\right) / B_{x}\right]^{-1}$, and the Lundquist number $S$ is evaluated using the parallel Spitzer resistivity since the current is mainly parallel the background field $B_{x 0} \gg B_{\perp}$. In the case $\tau_{B}>0$, this equation leads to an increase of the current sheet aspect ratio with respect to the Sweet-Parker scaling $\left(\delta / \Delta \approx S^{-1 / 2}\right)$.

In Fig. 7, we compare predictions from Eq. (4) with the experimental aspect ratio $\delta / \Delta$ measured during the phase $8 \mu \mathrm{s}<t<13 \mu \mathrm{s}$ in which the current sheet is clearly formed. The gray box indicates our estimated experimental error in determining the current sheet aspect ratio. The squares show the Sweet-Parker prediction $\delta / \Delta=S^{-1 / 2}$ using the experimental value of $S$. The Lundquist number $S<10$ puts these data in a collisional regime. The circles show values of $\delta / \Delta$ from

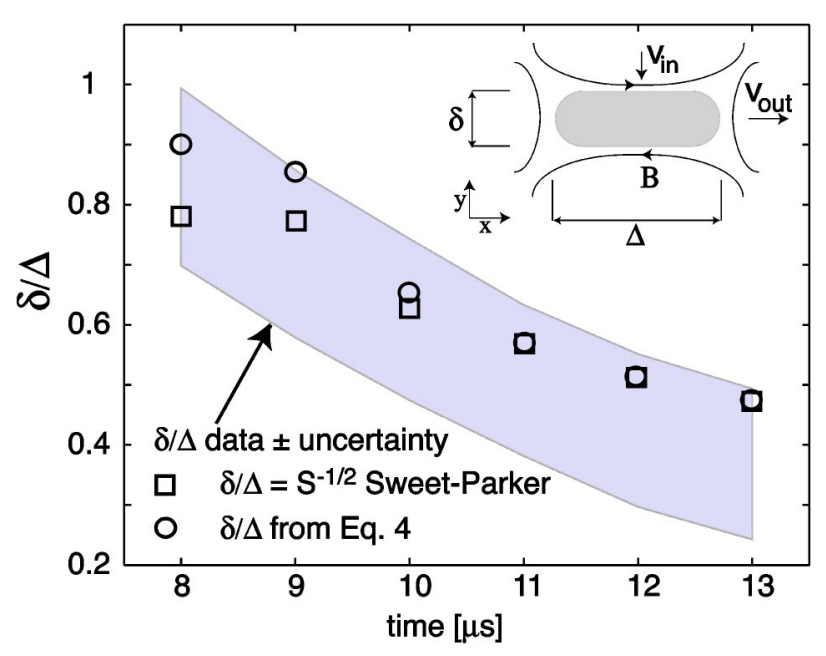

FIG. 7. Comparison of experimental aspect ratio $\delta / \Delta$ with predictions from Eq. (4) including (circles) and neglecting (squares) the contribution from $\tau_{B}$. The case $\tau_{B} \rightarrow \infty$ in Eq. (4) corresponds to the original Sweet-Parker model. The gray box indicates our estimated experimental error in determining the current sheet aspect ratio. The inset shows the Sweet-Parker geometry.

Eq. (4) retaining the contribution from $\tau_{B}$ where $B_{x}$ is evaluated at the edge of the current sheet. During this phase, the physics appears to be primarily 2D, and scales like a SweetParker current sheet.

It is interesting to note that for this narrow current sheet, the electron drift velocity for a Maxwellian distribution with $T_{e} \approx 3-4 \mathrm{eV}$ would need to be $V_{\mathrm{drift}} / v_{e} \approx 0.1$, which appears to exceed the estimated ion acoustic speed for hydrogen ions although the effect of current driven microinstabilities may be small in this collisional regime.

We remark that in a more collisionless regimes and plasma $\beta \approx 1$, previous experiments have shown that the current sheet width is determined by the ion gyroradius. ${ }^{10,26}$ In collisionless regimes and low $\beta$ plasmas, this dependence has not been observed. ${ }^{27}$

\section{Kink dynamics and 3D flux rope merging}

In this section, we show evidence for the coalescence of flux ropes during a fully $3 \mathrm{D}$ process in which the coalescence dynamics coexists with the kink mode dynamics. Late in time, the flux rope dynamics become less reproducible from shot to shot, so that single shot data acquisition becomes a more useful way to diagnose the plasma behavior. Here we use images of visible light emission, such as in Fig. 8 , which shows a time sequence of false color images (40200 ns gate time) of two flux ropes. The viewing perspective is end-on through a window at the chamber end, so that the plasma guns appear silhouetted in the foreground in Fig. 8(a). In Fig. 8(a), straight flux ropes are visible. A detailed study of the early phase $(t<15 \mu \mathrm{s})$ has been reported in Ref. 22 showing that the dynamics extracted from visible light images is in excellent agreement with measurements from in situ diagnostics.

Later, as $I_{\text {bias }}$ continues to increase, each rope begins to twist into a helical structure, Fig. 8(b). The time value does not represent the onset of this particular mode, but rather a period where such distinct structure is clearly visible. The 

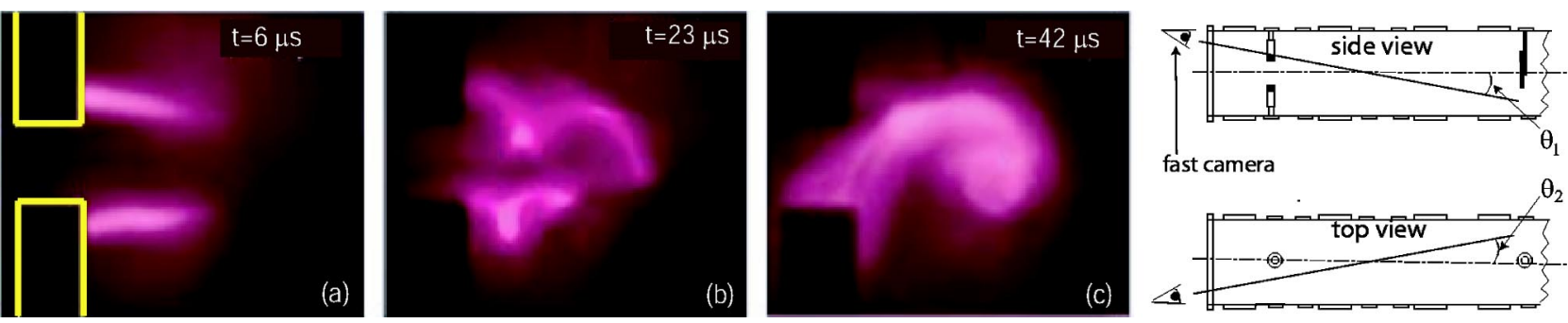

FIG. 8. (Color online) False color images of two magnetic flux ropes in hydrogen plasma reveal helically twisted structures (b,c). Times are given from the start of the applied current drive $(t=0)$. The silhouetted plasma guns in the foreground are outlined for early times (a). The viewing perspective of the fast camera (represented by an eye) is schematically shown by a continuous line in the side and top views of the RSX device. For clarity, the viewing perspective is artifically exaggerated in the figure. In the actual setup, $\theta_{1} \approx 1.5^{\circ}, \theta_{2} \approx 8^{\circ}$.

dominant azimuthal mode number of the helix is $m=1$ as determined from an external array of four equally spaced azimuthal magnetic coils. Preliminary magnetic measurements on a single flux rope suggest that the onset of the mode is consistent with the Kruskal-Shafranov ${ }^{25}$ limit for the external kink $2 \pi a B_{0 z} /\left[L B_{\perp}(r=a)\right]=1$, where $L$ is the length of the flux rope and $a$ is the radius where the selfgenerated azimuthal magnetic field $B_{\perp}$ is maximum.

As a consistency check, we note that the onset of helical structures corresponds to an increase in the overall inductance of the plasma/switching circuit. This should be generally true for flux $\Phi$ conserving motions in magnetic circuits with dissipated inductive energy $W=\frac{1}{2} L I^{2}=\Phi^{2} / 2 L{ }^{28}$ Figure $8(\mathrm{c})$, shows that at $t=43 \mu \mathrm{s}$ the two flux ropes remain distinct near the plasma guns but have merged into one single flux rope further down the axis of the RSX device. Density and temperature measurements at two different axial locations (not shown here) suggest that the coalescence process starts at the external anode and proceeds towards the gun like a zipper effect.

A quantitative analysis of the complex 3D physics of the coalescence process will require single-shot $3 \mathrm{D}$ diagnostics which are presently under construction, and will be the subject of future investigations.

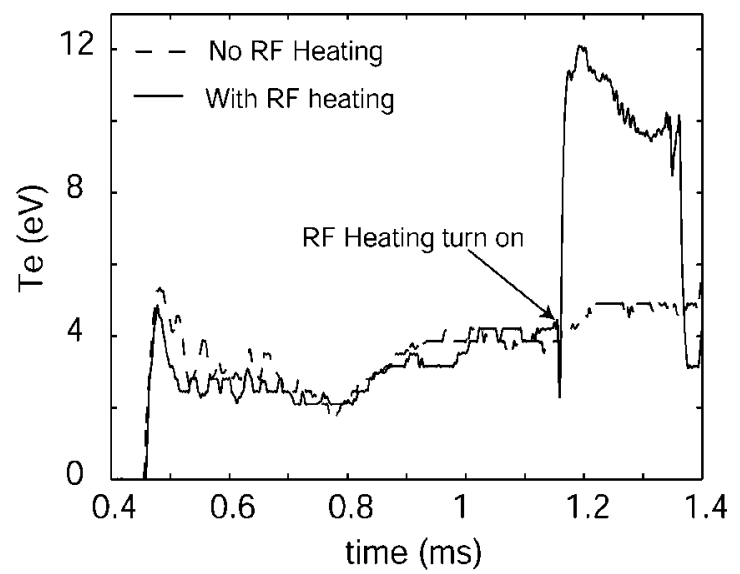

FIG. 9. Electron heating during a $300 \mathrm{~W}$ pulse (small fraction of this was actually coupled to the plasma) of rf power. The afterglow lasts over $20 \mu \mathrm{s}$, which allows a long rf free time interval with elevated $T_{e}$ and Lundquist number $\mathrm{S}$.

\section{FUTURE DIRECTIONS}

Presently, we are investigating the production of higher Lundquist number plasmas to access collisionless regimes. The Lundquist number depends strongly on the electron temperature $\left(S \propto T_{e}^{3 / 2}\right)$. A shorter column allows a longer kink stable time period and higher electron temperature. Radio frequency (rf) heating can also greatly increase the electron temperature. Figure 9 shows preliminary results from an experiment in which the flux rope goes through the aperture in a rf antenna. The electron temperature can be increased manyfold. For L mode scaling, ${ }^{29}$ which balances auxiliary heating with loss flows, the increase of $T_{e}$ scales as the square root of rf power. Therefore our existing $10 \mathrm{~kW}$ transmitter should increase $T_{e}$ shown in Fig. 9 by a factor $5-6$ over the $300 \mathrm{~W}$ case shown in Fig. 9. The elevated temperature lingers during an afterglow, when there is no rf to confuse the experimental interpretation.

In a collisionless regime and $\beta>m_{e} / m_{i}$. Ricci et al., have shown that magnetic reconnection signatures (such as the patterns of the electron density and of the out-of-plane magnetic field) are strongly influenced by the plasma $\beta$. One key attribute of RSX is the ability to continuously adjust plasma $\beta$ from $\beta \ll 1$ up to $\beta \approx 1$ by varying the axial magnetic field, independent of the plasma and current density. In future experiments, we will investigate the signatures of magnetic reconnection by varying the axial guide field in a collisionless regime. Moreover, experiments will be performed at guide field $B_{0 z}=1.5-2 \mathrm{kG}$, which would allow data at $\beta \leqslant m_{e} / m_{i} \approx 5 \times 10^{-4}$, where theoretical predictions indicate that qualitatively different physics dominate the reconnection dynamics. ${ }^{24}$

A comprehensive understanding of the dynamics of flux rope coalescence requires detailed 3D measurements at different axial positions along the device. These experiments are presently ongoing and preliminary measurements at two axial locations suggest that axial pressure gradient terms can be significant in the generalized Ohm's law.

\section{SUMMARY}

We presented first evidence of coalescence of magnetic flux ropes in a collisional plasma and in the presence of a strong guide field. Quasi-two-dimensional coalescence of 
two parallel flux ropes and the development of threedimensional merged structures are observed and studied in the RSX device.

During initial coalescence, a reconnection current sheet with a reversed induced current forms between the two flux ropes. The measured current sheet thickness is larger than the electron skin depth but smaller than the ion skin depth. Furthermore, the thickness does not vary for three different values of the strong external guide field. It is shown that the geometry of the observed current sheet is consistent with the Sweet-Parker model using a parallel Spitzer resistivity. The flux ropes eventually become kink unstable due to increasing current, and fast-gated camera images show the development of three-dimensional merged structures.

Several experimental improvements can facilitate access to more collisionless regimes and higher Lundquist numbers. The linear geometry simplifies analysis, interpretation, and modeling of experimental results. This capability allows us to investigate the effects of the axial guide field on magnetic reconnection.

\section{ACKNOWLEDGMENTS}

The authors gratefully acknowledge useful discussions with F. Porcelli, G. Fiksel, and J. M. Finn. This work was supported by Los Alamos National Laboratory, U.S. Department of Energy under Contract No. W-7405-ENG-36, and the Los Alamos Laboratory Directed Research and Development - Exploratory Research program.

${ }^{1}$ C. T. Russell, E. R. Priest, and L. C. Lee, Physics of Magnetic Flux Ropes, AGU Monograph 58 (AGU, Washington, D.C., 1990).

${ }^{2}$ R. M. Kulsrud, Phys. Plasmas 2, 1735 (1995).

${ }^{3}$ J. A. Slavin, R. P. Lepping, J. Gjerloev et al., Geophys. Res. Lett. 30, 14 (2003).

${ }^{4}$ R. Nakamura, W. Baumjohann, A. Runov et al., Geophys. Res. Lett. 29,
2140 (2002).

${ }^{5}$ S. A. Colgate, H. Li, and V. Pariev, Phys. Plasmas 8, 2425 (2001).

${ }^{6}$ P. P. Kronberg, Phys. Plasmas 10, 1985 (2003).

${ }^{7}$ I. Furno, C. Angioni, F. Porcelli et al., Nucl. Fusion 41, 403 (2001).

${ }^{8}$ S. C. Hsu and P. M. Bellan, Phys. Rev. Lett. 90, 215002 (2003).

${ }^{9}$ M. Yamada, Y. Ono, A. Hayakawa, M. Katsurai, and F. W. Perkins, Phys. Rev. Lett. 65, 721 (1990).

${ }^{10}$ M. Yamada, H. T. Ji, S. C. Hsu, T. Carter, R. Kulsrud, N. Bretz, F. Jobes, Y. Ono, and F. W. Perkins, Phys. Plasmas 4, 1936 (1997).

${ }^{11}$ M. R. Brown, Phys. Plasmas 6, 1717 (1999).

${ }^{12}$ C. D. Cothran, A. Falk, A. Fefferman, M. Landreman, M. R. Brown, and M. J. Schaffer, Phys. Plasmas 10, 1748 (2003).

${ }^{13}$ J. Hansen, S. K. P. Tripathi, and P. M. Bellan, Phys. Plasmas 11, 3177 (2004).

${ }^{14}$ W. Gekelman, J. E. Maggs, and H. Pfister, IEEE Trans. Plasma Sci. 20, 614 (1992).

${ }^{15}$ Y. Lau and J. Finn, Phys. Plasmas 3, 3983 (1996).

${ }^{16}$ D. Kondrashov, J. Feynman, P. C. Liewer, and A. Ruzmaikin, Astrophys. J. 519, 884 (1999).

${ }^{17}$ M. G. Linton R. B. Dahlburg, and S. K. Antiochos, Astrophys. J. 553, 905 (2001).

${ }^{18}$ I. Furno, T. Intrator, E. Torbert et al., Rev. Sci. Instrum. 4, 141 (2003).

${ }^{19}$ G. Fiksel, A. F. Almagri, D. Craig, M. Iida, S. C. Prager, and J. S. Sarff, Plasma Sources Sci. Technol. 5, 78 (1996).

${ }^{20} \mathrm{P}$. Sweet, Electromagnetic Phenomena in Cosmical Physics (Cambridge University Press, Cambridge, 1958).

${ }^{21}$ E. N. Parker, J. Geophys. Res. 62, 509 (1957).

${ }^{22}$ E. Hemsing, I. Furno, and T. Intrator, IEEE Trans. Plasma Sci. 33, 448 (2005).

${ }^{23}$ E. Hemsing, I. Furno, and T. Intrator, Rev. Sci. Instrum. 75, 4106 (2004).

${ }^{24}$ D. Biskamp, Magnetic Reconnection in Plasmas (Cambridge monographs on plasma physics, Cambridge, 2000).

${ }^{25}$ J. Freidberg, Ideal Magnetohydrodynamics (Modern Perspectives in Energy) (Plenum, New York, 1987).

${ }^{26}$ Y. Ono, A. Morita, M. Katsurai, and M. Yamada, Phys. Fluids B 5, 3691 (1993).

${ }^{27}$ J. Egedal, A. Fasoli, D. Tarkowski, and A. Scarabosio, Phys. Plasmas 8, 1935 (2001).

${ }^{28}$ P. M. Bellan, Spheromaks (Imperial College Press, London, 2000).

${ }^{29}$ J. Wesson, Tokamaks (Clarendon Press, Oxford, 2000).

${ }^{30}$ P. Ricci, J. U. Brackbill, W. Daughton, and G. Lapenta, Phys. Plasmas 11, 4102 (2004). 